\title{
The Application and Practice of Humanistic Care in the Emergency Department
}

\author{
Yuehuan $\mathbf{F u}^{*}$ \\ Department of Emergency, Fuzhou First hospital, Fu Zhou, Fu Jian 350009, China
}

\begin{abstract}
Objective: To study the application of humanized nursing in the emergency room, hence seek more suitable care model for emergency nursing. Methods: By reviewing the 521 patients visited to the hospital emergency department in April 2013 to May 2014, the patients were divided into control group and experimental group in accordance with the principle of voluntary and the principle of random. The control group has a total of 260 patients with normal nursing model. The experimental group has a total of 261 patients with the humanized nursing mode. Patients satisfaction degree was investigated after the nursing care. Results: The satisfaction questionnaire Results show that the satisfaction degree of experimental group patients was significantly higher than the control group patients $(p<0.05)$. Conclusion: Humanistic nursing can effectively improve the emergency department patients satisfaction degree, help to improve the quality of service, and has value for clinical promotion.
\end{abstract}

\author{
KEYWORDS \\ Humanized nursing \\ Emergency department \\ Clinical application
}

\section{Introduction}

The medical service is continuously improving rapidly with the development of modern medical technology. This is especially embodied in health care services. Modern nursing is a complex and systematic discipline, where it has a broad and strong applicability in health care services. The innovation of the modern nursing is not just about the innovation to the use of instruments and methods, more importantly is the humanistic care to patients. Human nature is the key in the traditional medical concept, where it focuses on care and comfort of people. The traditional medical concept in humanistic nursing becomes important in the emergency department where the task is important, time is tight, and doctor-patient relationship is tense [1]. In the hospital, the emergency department is an important external window, it not only act as main bear rescue of critically ill patients life, but adversely can also easy to occur medical disputes. In recent years, along with the social, medical development and patients' living standards that gradually improved, higher requirements for nursing ser-

Copyright $\odot 2016$ Yuehuan Fu

doi: 10.18686/jn.v5i3.9

Received: July 7, 2016; Accepted: August 2, 2016; Published online: August 17, 2016

This is an open-access article distributed under the terms of the Creative Commons Attribution Unported License (http://creativecommons.org/ licenses/by-nc/4.0/), which permits unrestricted use, distribution, and reproduction in any medium, provided the original work is properly cited. ${ }^{\star}$ Corresponding author: Fuzhou First hospital, Fu Zhou, Fu Jian Province, China 350009. E-mail: fuyuehuan123y@sina.com vice must also have to move forward. Humanization nursing was implemented in the emergency department in our hospital patients to meet the demand of patients, promote the harmonious relationship between nurses and patients and improve patient satisfaction to nursing service. The implementation has obtained a good effect.

\section{Data and methods}

\subsection{General data}

Five-hundred-and-twenty-one (521) patients who receive clinical treatment in our hospital emergency department were chosen from April 2013 to May 2014 as the research object. Through the principles of voluntarism and random distribution, 512 patients were randomly divided into two groups: control group and experimental group. The control group consist of 260 patients, 173 male patients and 87 female patients, age ranged from 0.5 to 83 , the average age is 54.2 , care mode is common nursing. While, the nursing group consisted of 261 patients, 162 male patients, 99 female patients, age ranged from 0.9 to 82 , average age is 52.1 , nursing way is humanized nursing. By contrast, patients in these two groups have no obvious difference on gender, age and disease $(p>0.05)$, where the differences were statistically significant.

\subsection{Nursing method}

2.2.1. Control group patients take routine nursing, specific measures

Patients who have respiratory dysfunction, the trache- 
otomy and mechanical assisted ventilation must be carried on. In addition, they still need routine anti-infection treatment. Nursing care was provided with carefully observation and records in the morning and evening. The patients were requested to fast and ban water in the early stage. Keep transfusion unobstructed and ensure early treatment go on wheels. Patients need absolute bed rest, make their head $30^{\circ}$ higher for promoting venous return and to avoid the phenomenon such as cerebral edema occur.

\subsubsection{Humanized nursing}

\section{Humanization of nursing methods}

Humanization of nursing care is a people-oriented nursing method for patients. The target for general nursing care is diseases but not patients, however, humanized nursing method treats the patients as the main target and patients' status was placed in the first place. Humanized nursing cares about patient feelings and understanding about the importance of nursing care. In particular, it is providing patients with more humanistic care in the process. Besides, the feelings of patients and their families were taken into consideration during the nursing care. It will not only eliminate the patient's concern, but at the same time it will also give full comfort and care to patients' families and hence achieve better nursing effect [2].

\section{Specification communication method}

In emergency care, patients are normally in critical condition making the doctor-patient relationship is more tensed. Since, the doctor-patient communication is tense and often short, it often causes serious consequences between doctor and patient because the small words misunderstanding. Therefore, standardized method of communication is particularly important. During nursing care, we need to use civilized terminology, do not be tense and use calm tone as much as possible during communication with patients and their families in order to ovoid patients think that they were in critical condition and to relax the patients' families. Particularly, we need to use more words which can put patients' families trust in the process, proper question and answer process between patient and family, do not apply vague statement, do not use the imperative tone, serve them with a smile and be considerate [3].

\section{Create a beautiful care environment}

In the patient's emergency treatment and recovery process, the environmental factor has very strong effect to the patient's psychological condition. A good environment will not only give physical and mental relaxation, relieve tension of the patients, it is also have very big improvement on emotions of patients' families. In addition, it also has a great role for medical workers in promoting the work since beautiful environment can keep them in calm to deal with related issues. Other than that, beautiful work environment also has a good effect on work enthusiasm of nursing staff. A beautiful environment not only needs renovation of hospital, but also needs arrangement of the nursing staff. All kinds of supplies must be kept neatly and rationally [4].

\section{Enrich the details of the humanized nursing}

During the process of specific nursing, details on nursing make the finishing point in nursing care. Many nursing problems and misunderstanding between doctor-patient is often due to the errors of mastery of detail in the process of nursing. Humanized nursing pays much attention to the details on patients' care, process of care and careful cares in all aspects in order to keep patients and their families in calm. In particular, we need to give patients appropriate touch, shake hands in the process of care, and give appropriate care for patients, can be a courtesy embrace or give patients' families a glass of warm water and others.

\section{Green channel}

The principle of "three first, one after" (check, treatment and hospitalization first, fees after) were adopted for the critically ill patients. If patients were hospitalized with special reasons such as being brought to the hospital after an accident without any of their family members, the staff should sign the relevant documents first and should be checked and given appropriate treatment before being prompted to make the payment. This is to ensure that the patients receive treatment within the shortest possible time. While needs to be checked, the hospital should provide special guidance to put an end to the disputes happened caused by patient who are not familiar with hospital environment and medical procedures, repeatedly rush about in the process of consultation, irritable mood and dissatisfaction caused by time delay [5].

\section{Psychological care}

Due to the differences in patients' geographical environment, folk custom, cultural level, living conditions and personal hygiene in emergency department, they will not have the same understanding on the aim of the treatment. So, the psychological factors for the treatment is complex and diverse such as anxiety, impatient, easily excited mood, especially unsatisfactorily with certain defects in the work of the hospital. Due to their high expectations, disappointment and anger produced by curative effect cannot be satisfied and they often abreact on health care workers. Therefore, provide excellent patient's psychological nursing is very important in the emergency work, medical staff should choose the appropriate language to comfort patients and patiently explain the illness to stabilize patients' mood. Try our best to help them get rid of negative emotions. Make them cooperate with the doctor that gives them the treatment to relieve the pain as soon as possible. Patiently explain the illness to the patients and their relatives, let the patients have a correct understanding on disease, give patient comfort and encouragement, make the patients get spiritual support to establish the confidence of conquer disease and face the disease with a positive at- 
Table 1. Patients satisfaction to nursing in emergency $(n=20)$.

\begin{tabular}{cccccc}
\hline Groups & Nursing methods & Way of communication & Nursing environment & Detail nursing & Total score \\
\hline Experimental group & $17.2 \pm 2.3$ & $17.9 \pm 2.1$ & $18.8 \pm 2.8$ & $19.2 \pm 3.0$ & $89.2 \pm 2.4$ \\
Control group & $16.5 \pm 2.1$ & $15.2 \pm 2.5$ & $14.8 \pm 2.6$ & $15.2 \pm 2.8$ & $72.5 \pm 3.1$ \\
\hline
\end{tabular}

Note: through statistical analysis, the two groups $p<0.05$, have statistical significance.

titude. In the implementation of the rescue, behavior should be gentle and elegant. Work actively but not in a mess, speak softly and cautiously. Win the understanding and respect, and gain trust with skilled technology, sincere attitude and great care for the patients. Medical personnel should tolerant on the extreme behavior of the patients, avoid any quarrel when communicating, be generous and friendly when being criticized by patients. Listen first, calmly think, have sympathy or understand them, then do a reasonable explanation. Pay attention to use plain, scientific, vivid and the language of comfort, avoid damaging boring language [6].

\subsection{Statistical methods}

Statistical software analysis processing (SPSS) 13.0 was used to analyze data in this experiment. Results were compared using chi-square, $p<0.05$ showed significantly statistical significance.

\section{Results}

By comparing the two groups, under the different environment of nursing, patients' recovery and satisfaction are relatively significant different, can fully reflect the advantage of humanistic care in this aspect. The investigation and analysis of patient satisfaction are shown in Table 1.

\section{Discussion}

By comparing the two groups, it is clearly show that the recovery and satisfaction of patients who treated by the humanized nursing were significantly better than routine care. This series of results clearly proved that the advantage of the humanized nursing and the importance of promoting it. At the same time, it is not difficult to draw the following conclusions.

\subsection{Harmonious relationship between nurses and patients}

Humanized service concept was integrated into the whole process of emergency treatment to meet the basic needs and wishes of patients. The patients will have warm and home feeling. Besides, the humanized service concept reflects the patients' right to know and consent. Patients will feel the humanistic care and concern from nurses. Other than that, it also greatly improves the communication and relationship between nurse and patient, thus improve the satisfaction of patients and their families for emergency nursing [7].

4.2. Improved the comprehensive quality nursing staff After the implementation of humanized nursing service, nurses' communication skills with patients have improved obviously. Innovation knowledge of nurses is also expanding. Nurses not only master the routine operation skills, but the related knowledge such as psychology, ethics, professional knowledge, practice, service concept and many other knowledge have been further improved, hence improving the overall quality.

\subsection{Changed the service concept}

In the conventional emergency treatment, the nurse just mechanically executes the doctor's advice and they lack of effective communication between nurses and patients. With the development of modern nursing, emergency nursing model has changed from pure technology into today's humanist care model as a whole. The changing of nurses concept by conducting "people-oriented" humanistic nursing service, will enhance the consciousness of nurses initiative serve the patients, hence improve the service quality. Practice has proved that the humanization nursing, is beneficial to the improvement of the nursing service quality and its continuous improvement.

Humanized care embodies the people-oriented concept fully. Through the nursing of the physiological and psychological for emergency patients, strive to improve medical environment, strengthen the communication with patients, give patients enough respect when nursing and maintain a good state of patients' mind to cooperate with treatment. Therefore, observation group has a higher satisfaction than the control group.

\section{References}

1. Bu H, Yan L, Chu J. The exploration and practice of high quality nursing service concept in the outpatient nursing. Chin General Pract Nurs. 2012;10(2):160-161.

2. Ni J, Wang $X$. The application of humanized nursing in emergency triage. China Medical Review. 2009;6:36-39.

3. Wang Y, Nie A. Brief talk of humanized service application in nursing work. Journal of Modern Medicine and Health Care. 2007;23:87-92.

4. Chang Q. The application of humanized nursing in emergency nursing work. Chinese Medical Emergency. 2007; 16:112-114.

5. Li X. The application of ADL assessment scale in the brain surgery humanized nursing service. J Hainan Med. 2012;23(18):145-146.

6. Han Y, Li H. The study of application effect of humanized nursing service in the emergency department. J Nurs Train. 2013;28(9):837-839.

7. Liu K, Gao Z. The influence of nurses pay-gain imbalance by carrying humanized nursing service activitie. Chin J Nurs. 2013;48(7):626-628. 